\title{
On a Divisibility Problem
}

\author{
Horst Alzer (*) - JózSEF SÁNdor (**)
}

ABSTRACT - We prove that there are no integers $n \geq 2$ and $k \geq 2$ such that $n^{k}$ divides $\varphi\left(n^{k}\right)+\sigma_{k}(n)$. For $k=2$ this settles a conjecture of Adiga and Ramaswamy.

Mathematics SubJect Classification (2010). 11A25.

KeYwords. Divisibility, Euler totient, sum of divisors, Weierstrass product, inequalities.

\section{Introduction}

We are concerned with the classical number theoretic functions $\varphi(n)$ and $\sigma_{\alpha}(n)$. If $n \geq 1$ is an integer, then $\varphi(n)$ denotes the number of positive integers not execeeding $n$ which are relatively prime to $n$. This function is known as the Euler totient. And, $\sigma_{\alpha}(n)$ denotes the sum of the $\alpha$ th powers of the divisiors of $n$. Here, $\alpha$ is a real or complex parameter. The main properties of these and other arithmetical functions can be found, for example, in [2].

Nicol [6] and Zhang [8] were the first who studied the divisibility problem

$$
n \mid(\varphi(n)+\sigma(n)) .
$$

Here, as usual, $\sigma=\sigma_{1}$. As each prime number $n$ satisfies relation (1), this has infinitely many solutions. Let $\omega(n)$ denote the number of distinct prime factors of $n$. If $\omega(n) \geq 2$, then the study of problem (1) is quite

(*) Indirizzo dell'A.: Morsbacher Str. 10, 51545 Waldbröl, Germany.

E-mail: H.Alzer@gmx.de

(**) Indirizzo dell'A.: Department of Mathematics, Babes-Bolyai University, 400084 Cluj, Romania.

E-mail: jsandor@math.ubbcluj.ro 
involved. Nicol showed that the solutions of (1) are not square-free and conjectured that they are all even. He also established that if $n=2^{k} \cdot 3 \cdot p$, where $p$ is a prime number of the form $p=2^{k-2} \cdot 7-1$ and $k \geq 2$ is an integer, then $n$ is a solution. Zhang proved that there are no solutions of the form $p^{a} \cdot q$, where $p$ and $q$ are distinct primes and $a$ is a positive integer. All cases $\omega(n)=2$ and $\omega(n)=3$ are settled in [5]. Also, in [5] the authors proved that for any fixed integer $n \geq 2$ there are only finitely many odd composite solutions with $\omega(n)=m$, where $m \geq 2$ is a fixed integer. They also obtained an asymptotic upper bound for the number of composite solutions.

Motivated by the methods and results published in [5], Harris [3], Yang [7], Jin and Tang [4] provided theorems for $\omega(n)=4$ as well as related results.

In 2008, Adiga and Ramaswamy [1] investigated an analogue of problem (1):

$$
n^{2} \mid\left(\varphi\left(n^{2}\right)+\sigma_{2}(n)\right)
$$

They proved that for any $n \geq 2$ and $\omega(n) \leq 3$ there is no solution. Moreover, they conjectured that there is no integer $n \geq 2$ satisfying (2).

In this note we study the following more general divisibility problem. Let $k \geq 2$ be a fixed integer. Do there exist integers $n \geq 2$ such that

$$
n^{k} \mid\left(\varphi\left(n^{k}\right)+\sigma_{k}(n)\right)
$$

is valid? In the next section, we show that the answer to this question is "no". For $k=2$ this settles the conjecture stated by Adiga and Ramaswamy.

\section{Lemmas and main Result}

In order to solve the divisibility problem (3) we need three auxiliary results. The first two lemmas offer properties of $\varphi$ and $\sigma_{k}$, whereas the third lemma provides an inequality involving the Weierstrass product $\prod_{j=1}^{n}\left(1-x_{j}\right)$.

LEMMA 1. Let $n \geq 2$ and $k \geq 2$ be integers. If (3) is solvable, then we have

$$
\varphi\left(n^{k}\right)+\sigma_{k}(n)=2 \cdot n^{k} .
$$


Proof. We obtain

$$
\sigma_{k}(n)=\sum_{d \mid n} d^{k}=\sum_{d \mid n}\left(\frac{n}{d}\right)^{k}=n^{k} \cdot \sum_{d \mid n} \frac{1}{d^{k}}
$$

and

$$
\sum_{d \mid n} \frac{1}{d^{k}} \leq \sum_{d \leq n} \frac{1}{d^{k}}<\sum_{d=1}^{\infty} \frac{1}{d^{k}}=\zeta(k),
$$

where $\zeta$ denotes the Riemann zeta function. Let

$$
A(n, k)=\frac{\varphi\left(n^{k}\right)+\sigma_{k}(n)}{n^{k}} .
$$

Since $\varphi\left(n^{k}\right)<n^{k}$, we get $A(n, k)<\zeta(k)+1 \leq \zeta(2)+1=2.64 \ldots$. On the other hand, using $\sigma_{k}(n)>n^{k}$ yields $A(n, k)>1$. Thus, $1<A(n, k)<3$. Since $A(n, k)$ is an integer, we conclude that (4) holds.

Lemma 2. For all integers $n \geq 2$ and $k \geq 2$ we have

$$
\frac{\sigma_{k}(n)}{n^{k}} \leq \frac{\sigma_{2}(n)}{n^{2}}<\prod_{p \mid n, p \text { prime }} \frac{1}{1-1 / p^{2}} .
$$

Proof. From (5) it follows that $\sigma_{k}(n) / n^{k}$ is decreasing with respect to $k$. This leads to the first inequality in (6). Let $n=\prod_{j=1}^{r} p_{j}{ }_{j}$ be the prime
factorization of $n$. Then,

$$
\frac{\sigma_{2}(n)}{n^{2}}=\prod_{j=1}^{r} \frac{p_{j}^{2 a_{j}+2}-1}{p_{j}^{2 a_{j}} \cdot\left(p_{j}^{2}-1\right)}=\prod_{j=1}^{r}\left(p_{j}^{2} \cdot \frac{1-1 / p_{j}^{2 a_{j}+2}}{p_{j}^{2}-1}\right)<\prod_{p \mid n} \frac{p^{2}}{p^{2}-1} .
$$

This settles the second inequality in (6).

Lemma 3. Let $x_{j} \in[0,1 /(j+1)]$ for $j=1, \ldots, r$. Then we have

$$
\prod_{j=1}^{r}\left(1-x_{j}\right)+\prod_{j=1}^{r}\left(1-x_{j}^{2}\right)^{-1} \leq 2 .
$$

The sign of equality holds if and only if $x_{1}=\ldots=x_{r}=0$.

Proof. We define

$$
F\left(x_{1}, \ldots, x_{r}\right)=\prod_{j=1}^{r}\left(1-x_{j}\right)+\prod_{j=1}^{r}\left(1-x_{j}^{2}\right)^{-1} .
$$


Moreover, let

$$
M=\left\{\left(x_{1}, \ldots, x_{r}\right) \in \mathbf{R}^{r} \mid 0 \leq x_{j} \leq 1 /(j+1)(j=1, \ldots, r)\right\}
$$

and

$$
\max _{\left(x_{1}, \ldots, x_{r}\right) \in M} F\left(x_{1}, \ldots, x_{r}\right)=F\left(c_{1}, \ldots, c_{r}\right) .
$$

It suffices to show that

$$
F\left(c_{1}, \ldots, c_{r}\right) \leq 2
$$

with equality if and only if $c_{1}=\ldots=c_{r}=0$.

We use induction on $r$. If $r=1$, then $0 \leq c_{1} \leq 1 / 2$ and

$$
2-F\left(c_{1}\right)=\frac{c_{1}}{1-c_{1}^{2}}\left(\frac{1}{2} \sqrt{5}+\frac{1}{2}+c_{1}\right)\left(\frac{1}{2} \sqrt{5}-\frac{1}{2}-c_{1}\right) \geq 0 .
$$

The sign of equality holds if and only if $c_{1}=0$.

Next, we assume that the assertion is true for $r-1$. We define for $j \in\{1, \ldots, r\}$ and $t \in[0,1 /(j+1)]$ :

$$
G(t)=F\left(c_{1}, \ldots, c_{j-1}, t, c_{j+1}, \ldots, c_{r}\right)
$$

Then,

$$
\max _{0 \leq t \leq 1 /(j+1)} G(t)=G\left(c_{j}\right)
$$

If $0<c_{j}<1 /(j+1)$, then there exists a number $\lambda \in(0,1)$ such that

$$
c_{j}=\lambda \cdot 0+(1-\lambda) \cdot \frac{1}{j+1} \text {. }
$$

Since

$$
G^{\prime \prime}(t)=\frac{6 t^{2}+2}{\left(1-t^{2}\right)^{3}} \prod_{i=1, i \neq j}^{r}\left(1-c_{i}^{2}\right)^{-1}>0,
$$

we conclude that $G$ is strictly convex on $[0,1 /(j+1)]$. Hence, we obtain

$$
\begin{gathered}
G\left(c_{j}\right)<\lambda G(0)+(1-\lambda) G(1 /(j+1)) \\
\leq \max \{G(0), G(1 /(j+1))\} \leq \max _{0 \leq t \leq 1 /(j+1)} G(t) .
\end{gathered}
$$

This contradicts (7). Thus,

$$
c_{j} \in\{0,1 /(j+1)\} \quad \text { for } \quad j=1, \ldots, r .
$$

We consider two cases. 
CASE 1. All numbers $c_{1}, \ldots, c_{r}$ are different from 0 .

Then, $c_{j}=1 /(j+1)(j=1, \ldots, r)$ and we get

$$
F\left(c_{1}, \ldots, c_{r}\right)=\frac{1}{r+1}+\prod_{j=1}^{r}\left(1+\frac{1}{j(j+2)}\right)=2-\frac{r}{(r+1)(r+2)}<2 .
$$

CASE 2. At least one of the numbers $c_{1}, \ldots, c_{r}$ is equal to 0 .

Let $c_{k}=0$ with $k \in\{1, \ldots, r\}$. We set

$$
\begin{gathered}
y_{j}=c_{j} \quad \text { for } \quad j=1, \ldots, k-1 \\
y_{j}=c_{j+1} \quad \text { for } \quad j=k, \ldots, r-1 .
\end{gathered}
$$

Then we have

$$
0 \leq y_{j} \leq \frac{1}{j+1} \quad \text { for } \quad j=1, \ldots, r-1 .
$$

Using the induction hypothesis gives

$$
F\left(c_{1}, \ldots, c_{r}\right)=F\left(y_{1}, \ldots, y_{r-1}\right) \leq 2
$$

with equality if and only if $y_{1}=\ldots=y_{r-1}=0$, that is, $c_{1}=\ldots=c_{k-1}=$ $c_{k+1}=\ldots=c_{r}=0$.

We are now in a position to prove our main result.

THEOREM. There are no integers $n \geq 2$ and $k \geq 2$ satisfying relation (3).

Proof. Using the known product representation $\varphi\left(n^{k}\right) / n^{k}=$ $\prod_{p \mid n}(1-1 / p)$ as well as Lemma 2 and the prime factorization $n=\prod_{j=1}^{r} p_{j} a_{j}$ we obtain

(8) $\frac{\varphi\left(n^{k}\right)}{n^{k}}+\frac{\sigma_{k}(n)}{n^{k}}<\prod_{p \mid n}\left(1-\frac{1}{p}\right)+\prod_{p \mid n} \frac{1}{1-1 / p^{2}}=\prod_{j=1}^{r}\left(1-x_{j}\right)+\prod_{j=1}^{r}\left(1-x_{j}^{2}\right)^{-1}$

with $x_{j}=1 / p_{j}$. Let $p_{1}<p_{2}<\cdots<p_{r}$. Then, $p_{j} \geq j+1$ for $j=1, \ldots, r$. Applying Lemma 3 reveals that the sum on the right-hand side of (8) is less than 2. Hence,

$$
\varphi\left(n^{k}\right)+\sigma_{k}(n)<2 \cdot n^{k} .
$$

From Lemma 1, we conclude that (3) has no solution.

Acknowledgments. We thank the referee for the careful reading of the manuscript. 


\section{REFERENCES}

[1] C. Adiga - H. N. Ramaswamy, A note on certain divisibility problem, Int. J. Math. Anal. 2 (2008), pp. 1157-1161.

[2] T. M. Apostol, Introduction to Analytic Number Theory, Springer, New York, 1976.

[3] K. HARRIS, On the classification of integers $n$ that divide $\varphi(n)+\sigma(n)$, J. Number Th. 129 (2009), pp. 2093-2110.

[4] Q.X. JIN - M. TANG, The 4-Nicol numbers having five different prime divisors, J. Integer Seq. 14 (2011), article 11.7.1 (electronic).

[5] F. LuCA - J. SÁndor, On a problem of Nicol and Zhang, J. Number Th. 128 (2008), pp. 1044-1059.

[6] C. A. NICOL, Some diophantine equations involving arithmetic functions, J. Math. Anal. Appl. 15 (1966), pp. 154-161.

[7] S. YANG, On a divisibility problem of Nicol and Zhang, (Chinese), Adv. Math. (China) 39 (2010), pp. 747-754.

[8] M. Zhang, On a divisibility problem. J. Sichuan Univ. Nat. Sci. Ed. 32 (1995), pp. 240-242.

Manoscritto pervenuto in redazione il 13 Luglio 2012. 